\title{
SQUARE-INTEGRABLE REPRESENTATIONS AND THE MACKEY THEORY
}

\author{
BY
}

TERJE SUND

\begin{abstract}
It is the purpose of this paper to clarify the relationship between the square-integrable irreducible representations of a 2nd countable unimodular locally compact group $G$ and a closed normal subgroup $N$ using the Mackey theory relating the dual spaces $\hat{G}$ and $\hat{N}$.
\end{abstract}

Introduction. In this paper $G$ will always denote a 2 nd countable locally compact unimodular group and $N$ a closed normal subgroup of $G$. We shall also assume that $N$ is of type I and regularly embedded in $G$ so that Mackey's theory applies. (See e.g. G. W. Mackey [8].) All representations will be continuous and unitary. We will use the following notation:

(1) $H_{\pi}=$ the Hilbert space for the representation $\pi$.

(2) $\hat{G}=$ the set of all equivalence classes of irreducible representations of $G$, also called the dual space of $G$.

(3) $\hat{G}_{s}=$ the set of all equivalence classes of irreducible square-integrable representations of $G$.

(4) $\theta^{G}=$ the representation of $G$ induced from the representation $\theta$ of $K$, where $K$ is a closed subgroup of $G$.

(5) $\pi_{1} \subseteq \pi$ means that the representation $\pi_{1}$ is a direct summand of the representation $\pi$.

(6) $\pi_{K}=$ the restriction of the representation $\pi$ of $G$ to the closed subgroup $K$.

(7) $\pi \simeq \omega$ means $\pi$ is unitarily equivalent to $\omega$.

(8) $G$ acts on $\hat{N}$ by inner automorphisms: $(g \cdot \omega)(n)=\omega\left(g^{-1} n g\right)$, where $g \in G, n \in N, \omega \in \hat{N}$.

We let $K_{\omega}=\{g \mid g \in G$ and $g \cdot \omega \simeq \omega\}=$ the stabilizer of $\omega \in \hat{N}$ under the action of $G$. ( $K_{\omega}$ is a closed subgroup of $G$ containing $N$, but need not be normal in $G$.)

(9) $G \cdot \omega=\{g \cdot \omega \mid g \in G\}=$ the $G$-orbit of $\omega \in \hat{N}$.

(10) $\hat{G}_{G \cdot \omega}=\left\{\pi \mid \pi \in \hat{G}\right.$ and $\left.\pi_{N} \simeq m \int_{G \cdot \omega}^{\oplus} g \cdot \omega d \mu(g \cdot \omega)\right\}$, where $m$ is a countable cardinal number depending on $\pi$ and $\mu$ some quasi-invariant measure on $N$ (also depending on $\pi$ ), see [8].

Received by the editors May 26,1972 and, in revised form, November 12, 1973.

AMS (MOS) subject classifications (1970). Primary 22D10, 22D30.

Key words and phrases. 2nd countable locally compact group, closed normal type I subgroup, regular embeddedness, continuous unitary representation, dual space, reduced dual, square-integrable irreducible representation, induced representation, action by inner automorphisms, orbits. 
(11) $V(\omega)=\left\{\theta \mid \theta \in \hat{K}_{\omega}\right.$ and $\theta_{n} \simeq m \cdot \omega$ for some countable cardinal number $m$ depending on $\theta$.

We will of ten denote a representation with the same symbol as its equivalence class. Note that $\pi \in \hat{G}_{s}$ if and only if there exist nonzero elements $h$ and $f$ in $H_{\pi}$ such that the function $g \mapsto(\pi(g) h, f)$ is in $L^{2}(G)$ (w.r.t. left Haar measure on $G$ ). It is equivalent to require that $g \mapsto(\pi(g) f, h)$ be in $L^{2}(G)$, for all $f, h \in H_{\pi}$, or that $\pi$ be an irreducible direct summand of the regular representation of $G$ on $L^{2}(G)$, (M. A. Rieffel [9]). For convenience we will now state the theorem due to Mackey which is the point of departure for our discussion.

Theorem (Mackey). (a) Let $\omega \in \hat{N}$ be fixed. Then the map

$$
\theta \mapsto \theta^{G}, \quad V(\omega) \rightarrow \hat{G}_{G \cdot \omega}
$$

is a bijection.

(b) Every $\pi \in \hat{G}$ belongs to $\hat{G}_{G \cdot \omega}$ for some $\omega \in \hat{N}$.

( $N$ is assumed to be of type I and regularly embedded in $G$.)

In particular, the theorem says that in order to survey the irreducible representations of $G$ it suffices to choose one $\omega$ from each orbit in $\hat{N}$ and use the maps $\theta \mapsto \theta^{G}$.

The exposition is organized in the following way. In $\$ 1$ we prove some general results about the relation between square-integrable representations of a group and its closed subgroups. The main result is Theorem (1.3) which gives necessary and sufficient conditions for a $\pi \in \hat{G}_{s}$ to be associated with a $G$-orbit in $\hat{N}$ with only square-integrable elements. In $\S 2$ we show that if $K_{\omega} / N$ is compact and $\omega \in \hat{N}_{s}$ then the fibre $\hat{G}_{G \cdot \omega}$ over $\omega$ is contained in $\hat{G}_{s}$. In $\S 3$ it is shown that $\hat{G}_{s}=\varnothing$ whenever $G$ has a noncompact closed central subgroup. As a corollary we obtain the well-known result that $\hat{G}_{s}=\varnothing$ when $G$ is a simply connected nilpotent analytic group. Finally, we show how to find the elements of $\hat{G}_{s}$ when the reduced dual $\hat{N}_{r}$ of $N$ is countable.

We would like to thank Johan F. Aarnes for leading us into the subject, and Siegfried Grosser for the many suggestions and remarks he has made about the presentation.

1. General results. In this section we find necessary and sufficient conditions for a $\pi \in \hat{G}_{s}$ to be in $\hat{G}_{G \cdot \omega}$, for an $\omega \in \hat{N}_{s}$, under the hypothesis of Mackey's theorem. We also provide an example where $\hat{N}_{s}=\varnothing$ but where the regular representation of $G$ has irreducible direct summands. Finally we show that if $\rho$ is an integrable representation of a closed subgroup $K$ of $G$ then the induced representation $\rho^{G}$ is integrable. First we need some lemmas about squareintegrable representations.

(1.1) Lemma. Let $G$ be $a$ 2nd countable locally compact group and $K$ a closed subgroup of $G$. Assume also that the left coset space $G / K$ has a $G$-invariant measure v. If $\pi \in \hat{G}_{s}$ and $\theta \in \hat{K}$ is a direct summand of $\pi_{K}$, then $\theta \in \hat{K}_{s}$. 
Proof. Let $f \neq 0$ be an element of $H_{\theta}\left(\subseteq H_{\pi}\right)$, then

$$
\begin{aligned}
+\infty & >\int_{G}|(\pi(g) f, f)|^{2} d \lambda(g)=\int_{G / K} \int_{K}|(\pi(g k) f, f)|^{2} d \mu(k) d \nu(g K) \\
& =\int_{G / K} \int_{K}\left|\left(\theta(k) f, \pi\left(g^{-1}\right) f\right)\right|^{2} d \mu(k) d \nu(g K) .
\end{aligned}
$$

(Here $\mu$ denotes a suitably normalized left Haar measure on $K$.) Hence

$$
\int_{K}\left|\left(\theta(k) f, \pi\left(g^{-1}\right) f\right)\right|^{2} d \mu(k)
$$

exists for $\nu$-almost all cosets $g K \in G / K$. Let $E$ be a $\nu$-measurable set in $G / K$ such that (*) is positive, for all $g K \in E$, and such that $\nu(E)>0$. Then choose $g K \in E$ such that (*) is finite. If $P$ is the orthogonal projection of $H_{\pi}$ onto $H_{\theta}$ then

$$
\left|\left(\theta(k) f, \pi\left(g^{-1}\right) f\right)\right|^{2}=\left|\left(\theta(k) f, P \pi\left(g^{-1}\right) f\right)\right|^{2}
$$

for all $k \in K$. Therefore

$$
0 \neq \int_{K}|(\theta(k) f, h)|^{2} d \mu(k)<+\infty,
$$

where $h=P \pi\left(g^{-1}\right) f \in H_{\theta}$. Since $\theta$ is irreducible it follows that $\theta \in \hat{K}_{s}$.

(1.2) Lemma. Let $N$ be a closed normal subgroup of $G$ and let $G$ act on $\hat{N}$ by inner automorphisms. If $\omega \in \hat{N}_{s}$ then the $G$-orbit $G \cdot \omega \subseteq \hat{N}_{s}$.

Proof. Let $\mu$ be a left Haar measure on $N$, and assume $f \in H_{\omega}, f \neq 0$. Then, for $g \in G$,

$$
\begin{aligned}
\int_{N}|((g \cdot \omega)(n) f, f)|^{2} d \mu(n) & =\int_{N}\left|\left(\omega\left(g^{-1} n g\right) f, f\right)\right|^{2} d \mu(n) \\
& =\int_{N}|(\omega(n) f, f)|^{2} d \mu\left(g n g^{-1}\right) \\
& =\int_{N}|(\omega(n) f, f)|^{2} d \mu_{g}(n),
\end{aligned}
$$

where $\mu_{g}: E \mapsto\left(g E g^{-1}\right)$ is left invariant on $N$. Hence there exists a function $\beta>0$ such that $\mu_{g}=\beta(g) \mu$. We conclude that $g \cdot \omega$ is square-integrable.

We next give necessary and sufficient conditions for an element $\pi$ of $\hat{G}_{s}$ to be associated with the orbit of an element $\omega \in \hat{N}_{s}$.

(1.3) Theorem. Let $G$ be $a$ 2nd countable locally compact group and $\pi \in \hat{G}_{s}$. Assume that $N$ is a closed normal subgroup of $G$ which is of type I and regularly embedded in $G$. Let $\omega \in \hat{N}$ be such that $\pi \in \hat{G}_{G \cdot \omega}$. Then the following conditions are equivalent.

(1) $\omega \in \hat{N}_{s}$.

(2) $\theta \subseteq \pi_{K_{\omega}}$, where $\theta \in V(\omega)$ and $\theta^{G} \simeq \pi$.

(3) $\pi_{N} \simeq m \cdot \bigoplus_{G \cdot \omega} g \cdot \omega$, where $m \in\left\{1,2,3, \ldots, \aleph_{0}\right\}$.

(4) The $G$-orbit $G \cdot \omega$ is countable. 
Proof. (1) $\Leftrightarrow$ (2): If $\pi \simeq \theta^{G}$ and $\theta \subseteq \pi_{K_{\omega}}$ then $\theta_{N} \subseteq \pi_{N}$. Hence $\omega \subseteq \theta_{N} \subseteq \pi_{N}$. Since $G / N$ is a locally compact group it has a $G$-invariant measure so that we may use Lemma (1.1) to conclude that $\omega \in \hat{N}_{s}$.

Conversely, if $\omega \in \hat{N}_{s}$, we have $G \cdot \omega \subseteq \hat{N}_{s}$, by Lemma (1.2). We now note that $L^{2}(N)$ is a Hilbert space of countable dimension so that the set $\hat{N}_{s}$ is countable, since every element of $\hat{N}_{s}$ has a representative which is a direct summand of the left regular representation of $N$ on $L^{2}(N)$. Hence $G \cdot \omega$ is countable. Next we observe that the map $g \cdot \omega \mapsto g K_{\omega}, G \cdot \omega \rightarrow G / K_{\omega}$ is a bijection, so that $G / K_{\omega}$ is countable. Then $\lambda\left(K_{\omega}\right)>0$, where $\lambda$ is a (left) Haar measure on $G$. It is then easy to verify that the map $U: f \mapsto f(e)$ from the subspace of $H_{\theta^{G}}$ generated by the functions whose supports are contained in $K_{\omega}$, gives a unitary equivalence between $\theta$ and the subrepresentation of $\left(\theta^{G}\right)_{K_{\omega}}$ on this subspace.

(1) $\Leftrightarrow$ (3): If $\omega \in N_{s}$ then $\theta \subseteq \pi_{K_{\omega}}$, so that $\omega \subseteq \theta_{N} \subseteq \pi_{N}$. Conversely, if $\pi_{N} \simeq m \cdot \bigoplus_{G \cdot \omega} g \cdot \omega$, then $\omega \subseteq \pi_{N}$, and by Lemma (1.1) we conclude that $\omega \in \hat{N}_{s}$.

(1) $\Rightarrow$ (4): If $\omega \in \hat{N}_{s}$ then $G \cdot \omega \in \hat{N}_{s}$, by Lemma (1.2); hence $G \cdot \omega$ is countable.

(4) $\Rightarrow(3)$ : Let $G \cdot \omega$ be countable. Then the direct integral $\int_{G \cdot \omega}^{\oplus}(g \cdot \omega) d \mu(g \cdot \omega)$ reduces to a direct sum. Thus $\pi_{N} \simeq m \cdot \bigoplus_{G \cdot \omega}(g \cdot \omega)$.

(1.4) Example. One may ask if every $\pi \in \hat{G}_{s}$ comes from a representation $\omega \in \hat{N}_{s}$. This is not the case as is seen by considering the following unimodular group $G$. Let $G_{1}$ be the "Heisenberg group" consisting of all real 3 by 3 matrices of the form

$$
\left(\begin{array}{lll}
1 & x & z \\
0 & 1 & y \\
0 & 0 & 1
\end{array}\right)
$$

Let $G=G_{1} / C$ where $C$ is the central subgroup

$$
\left\{\left(\begin{array}{ccc}
1 & 0 & n \\
0 & 1 & 0 \\
0 & 0 & 1
\end{array}\right) \ln \in \mathbf{Z}\right\} .
$$

$G$ is the semidirect product of $H$ and $N$ where

$$
H=\left\{\left(\begin{array}{lll}
1 & x & 0 \\
0 & 1 & 0 \\
0 & 0 & 1
\end{array}\right) \mid x \in \mathbf{R}\right\}
$$

and

$$
N=\left\{\left(\begin{array}{ccc}
1 & 0 & z \\
0 & 1 & y \\
0 & 0 & 1
\end{array}\right) \mid z, y \in \mathbf{R}\right\} / C
$$


Hence $H \simeq \mathbf{R}$ and $N \simeq \mathbf{R} \times \mathbf{T}$. The normal subgroup $N$ is seen to be regularly embedded in $G$, and the stability groups are: $K_{\alpha}=N$ for $\alpha \neq 1$ and $K_{1}=G$. Hence $G$ consists of all the induced $\alpha^{G}, \alpha \in \hat{N}, \alpha \neq 1$, together with the characters of $G / N$ lifted to $G$. The infinite dimensional representations $\alpha^{G}$ are known to be square-integrable, but no $\alpha$ is so, being a character of the noncompact $N$. Note that $G$ is a connected nilpotent Lie group but it is not simply connected, compare with Corollary (3.3).

In the example above we observe that the normal subgroup $N \simeq \mathbf{R} \times \mathbf{T}$ has measure 0 in $G$. If, on the contrary, we assume $\lambda\left(K_{\omega}\right)>0$ for some $\omega \in \hat{N}-\hat{N}_{s}$, we may conclude that $\theta \subseteq \pi_{K_{\omega}}$, for each pair $\pi, \theta\left(\pi \in \hat{G}_{G \cdot \omega}\right.$ and $\left.\theta \in V(\omega)\right)$ such that $\theta^{G} \simeq \pi$. (See e.g. the argument in the proof of (1) $\Leftrightarrow$ (2) of Theorem (1.3).) Hence it follows that $\omega \subseteq \pi_{N}$ so that $\omega$ is not square-integrable (Lemma (1.1)). Therefore we have:

(1.5) Proposition. Let $N$ be of type I and regularly embedded in $G$ (as in Mackey's theorem). Assume $\lambda\left(K_{\omega}\right)>0$ for every $\omega \in \hat{N}_{s}$. Then the following holds.

(i) If $\hat{N}_{s}=\varnothing$ then $\hat{G}_{s}=\varnothing$.

(ii) If $\pi \in \hat{G}_{s}, \pi \in \hat{G}_{G \cdot \omega}$; then $\omega \in \hat{N}_{s}$.

We shall now investigate the behavior of integrable representations under the inducing process. We would like to thank Professor J. Gil de Lamadrid for pointing out an error in the proof of the original version of the following result.

(1.6) Proposition. Let $K$ be a closed subgroup of $G$, and assume $K$ and $G$ both are unimodular. If $\rho$ is a cyclic integrable representation of $K($ i.e. $k \mapsto(\rho(k) \xi, \xi)$ is in $L^{1}(K)$ for some cyclic vector $\xi$ of $\left.\rho\right)$, then the cyclic representation $\pi=\rho^{G}$ is integrable.

Proof. Let $\rho$ be a cyclic representation of $K$. Then $\pi=\rho^{G}$ is cyclic. Since $\rho$ is cyclic it may be constructed from a measure $\mu$ of positive type on $K$ in the usual way. It is well known that the induced representation $\pi=\rho^{G}$ is unitarily equivalent with the representation constructed from the induced measure $\nu$ on $G$ [4]. Furthermore, it is shown in [4] that for 2 nd countable groups $G$, the representation constructed from a given measure of positive type is always cyclic. Hence $\rho^{G}$ is cyclic.

Let $\nu$ be a measure of positive type on $G$ corresponding to $\pi=\rho^{G}$. If $f, h \in C_{c}(G)=$ the *algebra of continuous functions with compact support on $G$ with the usual convolution and involution, we have $(\pi(x)[f],[h])=\nu\left(h^{*} * f_{x}\right)$, where $[f]=f+N, N=\left\{f \mid \nu\left(f^{*} * f\right)=0, f \in C_{c}(G)\right\}$, and $f_{x}(y)=f\left(x^{-1} y\right)$; $x, y \in G$. Let $\xi$ be a cyclic vector for $\rho$. Then $h \mapsto \mu(h)=\int_{K} h(k)(\rho(k) \xi, \xi) d k$, $h \in C_{c}(K)$, is a measure of positive type which gives rise to $\rho$. The induced measure $\tilde{\mu}$ is given by $\tilde{\mu}(h)=\mu(h \mid K)$ (since $G$ and $K$ are unimodular by hypothesis). Since the induced measure $\tilde{\mu}$ gives rise to $\pi=\rho^{G}$, we may assume $\nu=\tilde{\mu}$. Hulanicki has shown that there exists $f \in C_{c}(G)$ such that $[f]$ is a cyclic vector for $\pi$ [Proc. Amer. Math. Soc. 38 (1973), 220]. We choose such an $f \in$ $C_{c}(G)$. Then 


$$
\begin{aligned}
(\pi(x)[f],[f])_{\nu} & =\nu\left(f^{*} *\left(f_{x}\right)\right)=\mu\left(\left(f^{*} * f_{x}\right) \mid K\right) \\
& =\int_{K}\left(f^{*} * f_{x}\right)(k)(\rho(k) \xi, \xi) d k \\
& =\int_{K} \int_{G} f^{*}(y) f\left(x^{-1} y^{-1} k\right)(\rho(k) \xi, \xi) d y d k .
\end{aligned}
$$

Using Fubini's theorem and unimodularity we derive,

$$
\begin{aligned}
\int_{G}\left|(\pi(x)[f],[f])_{V}\right| d x & \leq \int_{G} \int_{K} \int_{G}\left|f^{*}(k y) f\left(x^{-1} y^{-1}\right)(\rho(k) \xi, \xi)\right| d y d k d x \\
& =\int_{K} \int_{G} \int_{G}\left|f^{*}(k y) f\left(x^{-1} y^{-1}\right)(\rho(k) \xi, \xi)\right| d x d y d k \\
& =\int_{K} \int_{G} \int_{G}\left|f^{*}(k y) f\left(x^{-1}\right)(\rho(k) \xi, \xi)\right| d x d y d k \\
& =\int_{G}\left|f\left(x^{-1}\right)\right| d x \int_{K} \int_{G}\left|f^{*}(y)(\rho(k) \xi, \xi)\right| d y d k \\
& =\|f\|_{1}^{2} \int_{K}|(\rho(k) \xi, \xi)| d k<+\infty .
\end{aligned}
$$

Hence $\pi=\rho^{G}$ is integrable.

2. The case " $K_{\omega} / N$ compact." Starting with a representation $\omega \in \hat{N}_{s}$ we shall now find a sufficient condition for $\hat{G}_{G \cdot \omega}$, the fibre over $\omega$, to be contained in $\hat{G}_{\boldsymbol{s}}$. The situation is as in Mackey's theorem.

(2.1) Proposition. Let $\omega \in \hat{N}_{s}$ and assume that $K_{\omega} / N$ is compact. Then $\hat{G}_{G \cdot \omega}$ $\subseteq \hat{G}_{s}$. In particular, each orbit $G \cdot \omega$ in $\hat{N}_{s}$ corresponds to a fibre $\hat{G}_{G \cdot \omega}$ in $\hat{G}_{s}$.

Proof. Let $\pi \in \hat{G}_{G \cdot \omega}$. Then $\pi \simeq \theta^{G}$, for some $\theta \in V(\omega)$. It suffices to show that $\theta$ is square-integrable since a direct summand of the regular representation of $K_{\omega}$ will give a direct summand of the regular representation of $G$ when we induce it to $G$. Let $f \neq 0$ be an element of $H_{\omega}\left(\subseteq H_{\theta}\right)$. Then, if $k \in K_{\omega}$ and $P: H_{\theta} \rightarrow H_{\omega}$ is the orthogonal projection,

$$
\begin{aligned}
\int_{N}|(\theta(k n) f, f)|^{2} d \gamma(n) & =\int_{N}\left|\left(\theta(n) f, \theta\left(k^{-1}\right) f\right)\right|^{2} d \gamma(n) \\
& =\int_{N}\left|\left(\omega(n) f, P \theta\left(k^{-1}\right) f\right)\right|^{2} d \gamma(n) \\
& =d(\omega)^{-1}\|\omega(n) f\|^{2}\left\|P \theta\left(k^{-1}\right) f\right\|^{2} \leq d(\omega)^{-1}\|f\|^{4} .
\end{aligned}
$$

Here, $d(\omega)$ denotes the formal dimension of $\omega$, and the last equality follows from the orthogonality relations for square-integrable representations (see J. Dixmier [2]). $\gamma$ denotes a left Haar measure on $N$. Hence we have

$$
\begin{aligned}
\int_{K_{\omega}}|(\theta(k) f, f)|^{2} d \mu(k) & =\int_{K_{\omega} N} \int_{N}|(\theta(k n) f, f)|^{2} d \gamma(n) d \nu(k N) \\
& \leq \int_{K_{\omega} / N} d(\omega)^{-1}\|f\|^{4} d \nu(k N)=\nu\left(K_{\omega} / N\right) d(\omega)^{-1}\|f\|^{4}
\end{aligned}
$$


since $K_{\omega} / N$ is compact. Hence $\theta \in\left(K_{\omega}\right)_{s}^{\wedge}$ and therefore $\pi \in \hat{G}_{s}$.

(2.2) Corollary. If $\omega \in \hat{N}_{s}$ and $K_{\omega} / N$ is compact then $V(\omega) \subseteq\left(K_{\omega}\right)_{s}^{\hat{s}}$.

Proof. Apply Proposition (2.1), with $G=K_{\omega}$.

\section{Examples and applications.}

(3.1) Example. In Proposition (2.1), the hypothesis that $K_{\omega} / N$ be compact cannot be removed. This is seen, e.g., when we let $G=V \times H$ where $H$ is a 2 nd countable compact group, and $V$ is a vector group. If we first let $N=V, \hat{N}$ is isomorphic with $N$, so that the orbits are countably separated and therefore $N$ regularly embedded in $G$. We apply Mackey's theorem: If $\pi \in \hat{G}_{G \cdot \omega}$, we have $K_{\omega}=G$ since $G$ acts trivially on $\hat{N}$. Hence $\pi_{N} \simeq m \cdot \omega$, for some countable cardinal number $m$, and if $f \in H_{\omega}\left(\subseteq H_{n}\right), f \neq 0$, we obtain

$$
\begin{aligned}
\int_{G}|(\pi(g) f, f)|^{2} d \lambda(g) & =\int_{H} \int_{V}|(\pi(v, h) f, f)|^{2} d \nu(v) d \gamma(h) \\
& =\int_{H} \int_{V}\left|\left(\pi(\nu, e) f, \pi\left(e, h^{-1}\right) f\right)\right|^{2} d \nu(v) d \gamma(h) \\
& =\int_{H} \int_{V}\left|\left(\omega(v), \pi\left(e, h^{-1}\right) f\right)\right|^{2} d \nu(v) d \gamma(h)=+\infty,
\end{aligned}
$$

since the Haar measure $\lambda$ on the direct product $V \times H$ is the direct product of the Haar measures $\nu$ on $V$ and $\gamma$ on $H, \nu$ and $\gamma$ suitably normalized. (We also used the fact that $\omega$ is a character on $V$ and therefore not square-integrable, so that the inner integral is $+\infty$, for all $h$ in a set $E \subseteq H$, with $\gamma(E)>0$.) Hence $\hat{G}_{s}=\varnothing$. Next, if we let $N=H$, then $\hat{H}_{s}=\hat{H}$, so that the statement of Proposition (2.1) does not hold. In this case, $K_{\omega}=G$ so that $K_{\omega} / N \cong V$ is not compact, $\omega$ being an arbitrary element of $\hat{N}$. Observe that $H$ is regularly embedded since $\hat{H}=\hat{H}_{s}$ is countable and also discrete, and the orbits are the one point sets.

From the first part of this example we now derive the following result, the proof of which is a slight modification of the one given above for $V \times H$.

(3.2) Proposition. Let $G$ be a 2nd countable locally compact group having a noncompact closed subgroup $N$ contained in its center, then $\hat{G}_{s}=\varnothing$.

Proof. We only note that $\pi_{N} \simeq m \cdot \omega$, if $\pi \in \hat{G}_{G \cdot \omega}$ and that $G / N$ has an invariant measure $\gamma$. Thus we get, for $f \in H_{\omega}\left(\subseteq H_{\pi}\right), f \neq 0$,

$$
\begin{aligned}
0 & \neq \int_{G}|(\pi(g) f, f)|^{2} d \lambda(g)=\int_{G / N} \int_{N}|(\pi(g n) f, f)|^{2} d \nu(n) d \gamma(g) \\
& =\int_{G / N} \int_{N}\left|\left(\omega(n) f, \pi\left(g^{-1}\right) f\right)\right|^{2} d \nu(n) d \gamma(g N)=+\infty .
\end{aligned}
$$

(Note that $\omega \in \hat{N}$ is not square-integrable since it is a character of the noncompact group $N$.) 
As a corollary we obtain the following well-known result.

(3.3) Corollary. If $G$ is a simply connected nilpotent analytic group then $G_{s}=$ $\varnothing .\left({ }^{1}\right)$

Proof. From the definition of nilpotence we see that $G$ contains a nontrivial closed central subgroup $N$. This subgroup is also simply connected and hence a vector group, i.e., isomorphic with $\mathbf{R}^{n}$, for some $n$ (see Hochschild [5]). The orbits in $\hat{N}$ under the action of $G$ are the one-point sets in $\hat{N} \cong \mathbf{R}^{n}$, so that $N$ is regularly embedded. By Proposition (3.2), $\hat{G}_{s}=\varnothing$.

Since the Plancherel measure is concentrated on the reduced dual space $\hat{N}_{r}$ of $N$ it follows that whenever $\hat{N}_{r}$ is countable the regular representation $\rho$ of $N$ decomposes into a direct sum of irreducible subrepresentations: $\rho=\bigoplus_{i}\left(m_{i} \cdot \omega_{i}\right)$, $\omega_{i} \in \hat{N}_{r}$ (see J. Dixmier [2, 18.8.1-4]).

This occurs, e.g., when $N$ is compact. On the other hand, there are examples of noncompact groups $N$ such that $\hat{N}_{r}$ is countable.(L. Baggett [1]. In [1] it is also shown that if $N$ is a separable Lie group with countable reduced dual then $N$ is compact.) We will now construct the square-integrable representations of $G$ when $\hat{N}_{r}$ is countable.

(3.4) Lemma. Let the hypothesis be as in Mackey's theorem and assume that, in addition, $\hat{N}_{r}$ is countable. If $\pi \in \hat{G}_{s}$ then $\pi_{N} \simeq m \cdot \bigoplus_{G \cdot \omega}(g \cdot \omega)$, for some $\omega \in \hat{N}_{s}$.

Proof. From a generalization of the Frobenius reciprocity theorem due to Mackey (see G. W. Mackey [7]) it follows that $\pi_{N} \simeq \bigoplus_{i} m_{i} \cdot \omega_{i}$, where $\omega_{i} \in \hat{N}_{s}$ and $m_{i}$ is a countable cardinal number. On the other hand by Mackey's theorem we also have, for some $\omega \in \hat{N}$,

$$
\pi_{N} \simeq m \cdot \int_{G \cdot \omega}^{\oplus}(g \cdot \omega) d \mu(g \cdot \omega), \quad \text { where } m \leq \aleph_{0} .
$$

Hence, and since the decomposition in (*) is the essentially unique central decomposition of $\pi_{N}$ (see J. Dixmier [2] and G. W. Mackey [8]), we may conclude that the two decompositions of $\pi_{N}$ coincide.

(3.5) Theorem. Let the hypothesis be as in Mackey's theorem and assume that, in addition, $\hat{N}_{r}$ is countable. Then the following holds.

(i) If $\omega \in \hat{N}_{s}$ then the map $\theta \mapsto \theta^{G}$ of $V(\omega) \cap\left(K_{\omega}\right)_{s}$, into $\hat{G}_{G \cdot \omega} \cap \hat{G}_{s}$ is a bijection.

(ii) If $\pi \in \hat{G}_{s}$, then $\pi$ may be obtained in the form $\pi \simeq \theta^{G}$ where $\theta \in V(\omega)$ $\cap\left(K_{\omega}\right)_{s}^{\wedge}$ for some $\omega \in \hat{N}_{s}$.

Note. $\hat{G}_{s}$ may, of course, be empty (see, e.g., Example (3.1)). However, the theorem tells us that if $\hat{G}_{s}=\varnothing$ then already $V(\omega) \cap\left(K_{\omega}\right)_{s}=\varnothing$.

(1) This result is known to hold, more generally, for simply connected nilpotent Lie groups. C. C. Moore communicates to the author that he has found necessary and sufficient conditions for nilpotent Lie groups to have square-integrable representations. 
Proof. If $\pi \in \hat{G}_{s}$ it follows from Lemma (3.4) that $\pi \in \hat{G}_{G \cdot \omega}$, for some $\omega \in \hat{N}_{s}$. If $\theta \in V(\omega)$ and $\theta^{G} \simeq \pi$ then $\theta \subseteq \pi_{K_{\omega}}$ (Theorem (1.3)). Now the $G$-orbit $G \cdot \omega$ is countable, so that $\lambda\left(K_{\omega}\right)>0$. Let $f \neq 0, f \in H_{\theta}\left(\subseteq H_{\pi}\right)$. Then

$$
\int_{K_{\omega}}|(\theta(k) f, f)|^{2} d \lambda(k)=\int_{K_{\omega}}|(\pi(k) f, f)|^{2} d \lambda(k) \leq \int_{G}|(\pi(g) f, f)|^{2} d \lambda(g)<+\infty .
$$

Hence $\theta \in V(\omega) \cap\left(K_{\omega}\right)_{s}^{\hat{s}}$, and (ii) follows.

If $\theta \in V(\omega) \cap\left(K_{\omega}\right)_{s}^{\hat{s}}$ then $\theta^{G} \in \hat{G}_{G \cdot \omega} \cap \hat{G}_{s}$, by Mackey's theorem and the fact that if $\theta \in\left(K_{\omega}\right)_{s}^{\wedge}$ then $\theta^{G}$ is a subrepresentation of the regular representation of G. Surjectivity of the map $\theta \mapsto \theta^{G}$ is demonstrated exactly as in the proof of (ii).

Note. Lemma (3.4) says that the case " $\hat{N}_{r}$ countable" is an instance where Theorem (1.3) holds. In fact, an analogous version of Theorem (3.5) may be proved whenever Theorem (1.3) holds. $\left({ }^{2}\right)$

\section{REFERENCES}

1. L. Baggett, A separable group having a discrete dual space is compact, J. Functional Analysis 10 (1972), 131-148.

2. J. Dixmier, Les $C^{*}$-algèbres et leurs représentations, Cahiers Scientifiques, fasc. 29, GauthierVillars, Paris, 1964. MR 30 \# 1404.

3. E. Effros and F. Hahn, Locally compact transformation groups and $C^{*}$-algebras, Mem. Amer. Math. Soc. No. 75 (1967). MR 37 \#2895.

4. F. Greenleaf and M. Moskowitz, Cyclic vectors for representations of locally compact groups, Math. Ann. 190 (1971), 265-288. MR 45 \#6978.

5. G. Hochschild, The structure of Lie groups, Holden-Day, San Francisco, 1965. MR 34 \#7696.

6. A. Kirillov, Unitary representations of nilpotent Lie groups, Uspehi Mat. Nauk 17 (1962), no. 4 (106), 57-110 = Russian Math. Surveys 17 (1962), no. 4, 53-104. MR 25 \#5396.

7. G. W. Mackey, Induced representations of locally compact groups. II. The Frobenius reciprocity theorem, Ann. of Math. (2) 58 (1953), 193-221. MR 15, 101.

8. The theory of group representations, Lecture Notes (Summer, 1955), Dept. of Math., Univ. of Chicago, Chicago, Ill. MR 19, 117.

9. M. Rieffel, Square-integrable representations of Hilbert algebras, J. Functional Analysis 3 (1969), 265-300. MR 39 \#6094.

10. A. Weil, L'intégration dans les groupes topologiques et ses applications, Hermann, Paris, 1940.

Department of Mathematics, University of Minnesota, Minneapolis, Minnesota 55455

Current address: Department of Mathematics, University of Maryland, College Park, Maryland 20742

(2) ADDED IN PROOF. The author would like to point out that A. Kleppner and R. Lipsman, The Plancherel formula for group extensions, Ann. Sci. École Norm. Sup. 5 (1972), $\S 11$, contains results related to the results of the present article. 